\title{
Spontaneous excitation of an accelerated hydrogen atom coupled with electromagnetic vacuum fluctuations
}

\author{
Zhiying $\mathrm{Zhu}^{2}$, Hongwei $\mathrm{Yu}^{1,2, *}$ and Shizhuan $\mathrm{Lu}^{2}$ \\ ${ }^{1}$ CCAST(World Lab.), P. O. Box 8730, Beijing, 100080, P. R. China \\ ${ }^{2}$ Department of Physics and Institute of Physics, \\ Hunan Normal University, Changsha, Hunan 410081, China ${ }^{\dagger}$
}

\begin{abstract}
We consider a multilevel hydrogen atom in interaction with the quantum electromagnetic field and separately calculate the contributions of the vacuum fluctuation and radiation reaction to the rate of change of the mean atomic energy of the atom for uniform acceleration. It is found that the acceleration disturbs the vacuum fluctuations in such a way that the delicate balance between the contributions of vacuum fluctuation and radiation reaction that exists for inertial atoms is broken, so that the transitions to higher-lying states from ground state are possible even in vacuum. In contrast to the case of an atom interacting with a scalar field, the contributions of both electromagnetic vacuum fluctuations and radiation reaction to the spontaneous emission rate are affected by the acceleration, and furthermore the contribution of the vacuum fluctuations contains a non-thermal acceleration-dependent correction, which is possibly observable.
\end{abstract}

\footnotetext{
* Corresponding author

$\dagger$ Mailing address
} 


\section{INTRODUCTION}

Spontaneous emission is one of the most important features of atoms and it may be attributed to vacuum fluctuations [1, 2], or radiation reaction [3], or a combination of them 4]. The ambiguity in physical interpretation arises from different choices of ordering of commuting operators of atom and field in a Heisenberg picture approach to the problem. The ambiguity was resloved, when Dalibard, Dupont-Roc and Cohen-Tannoudji showed [5, 6] that there exists a symmetric operator ordering that the distinct contributions of vacuum fluctuations and radiation reaction to the rate of change of an atomic observable are separately Hermitian and able to possess an independent physical meaning.

Recently, Audretsch, Müller and Holzmann have generalized the formalism of Ref. [6] to evaluate vacuum fluctuations and radiation reaction contributions to the spontaneous excitation rate [7] and radiative energy shifts 8] of an accelerated two-level atom interacting with a scalar field in a Minkowski vacuum. In particular, their results show that when the atom is accelerated, the delicate balance between vacuum fluctuations and radiation reaction is altered since the contribution of vacuum fluctuations to the rate of change of the mean excitation energy is modified while that of the radiation reaction remains the same. Thus transitions to excited states for ground-state atoms become possible even in vacuum. Based upon the formalism developed by Audretsch and Müller 7], the effects of modified vacuum fluctuations and radiation reaction due to the presence of a reflecting plane boundary upon the spontaneous excitation of both an inertial and a uniformly accelerated atom interacting with a quantized real massless scalar field have recently been discussed and similar conclusions are reached [9].

However, a two-level atom interacting with a scalar field is more or less a toy model, and a more realistic system would be a multi-level atom, a hydrogen atom, for instance, in interaction with a quantized electromagnetic field. Such a system was recently examined in terms of the radiative energy shifts of the accelerated atom [10] using the method of Ref. [8], where non-thermal corrections to the energy shifts were found in addition to the usual thermal ones associated with the temperature $T=a / 2 \pi$. To make the spectrum of research complete, the atom's spontaneous emission rate in the same realistic system will be considered in present paper following the general method of Ref. 7]. We will separately calculate the contribution of vacuum fluctuations and the radiation reaction to the rate of variation of the atomic energy of a hydrogen atom interacting with the quantum electromagnetic field. We will see that both the effects of vacuum fluctuations and radiation reaction on the atom are changed by the acceleration. This is in sharp contrast to the scalar field case where the contribution of radiation reaction is not altered by the acceleration. A dramatic feature is that the contribution of electromagnetic vacuum fluctuations to the spontaneous emission rate contains an extra non-thermal term proportional to $a^{2}$, the proper acceleration squared, in contrast to the scalar field case where the effect of acceleration is purely thermal. Therefore the equivalence between uniform acceleration and thermal fields is lost when the scalar field is replaced by the electromagnetic field as has been argued elsewhere in other 
different context [1].

\section{INTERACTION OF A HYDROGEN ATOM AND THE ELECTROMAGNETIC FIELD}

Let us now briefly review the model introduced in Ref. [10], where a linear interaction between a hydrogen atom and the quantum electrmagnetic field is assumed. The Hamiltonian that governs the time evolution of the atom with respect to the proper time $\tau$ is written as

$$
H_{A}(\tau)=\sum_{n} \omega_{n} \sigma_{n n}(\tau)
$$

where $|n\rangle$ denotes a series of stationary atomic states with energies $\omega_{n}$ and $\sigma_{n n}(\tau)=|n\rangle\langle n|$. The units in which $\hbar=c=1$ is adopted here and hereafter. The free Hamiltonian of the quantum electromagnetic field is

$$
H_{F}(\tau)=\sum_{k} \omega_{\vec{k}} a_{\vec{k}}^{\dagger} a_{\vec{k}} \frac{d t}{d \tau},
$$

where $\vec{k}$ denotes the wave vector and polarization of the field modes. We couple the hydrogen atom and the quantum electromagnetic field in the multipolar coupling scheme [10]

$$
H_{I}(\tau)=-e \mathbf{r}(\tau) \cdot \mathbf{E}(x(\tau))=-e \sum_{m n} \mathbf{r}_{m n} \cdot \mathbf{E}(x(\tau)) \sigma_{m n}(\tau)
$$

where $e$ is the electron electric charge, er the atomic electric dipole moment, $x(\tau) \leftrightarrow$ $(t(\tau), \mathbf{x}(\tau))$ the space-time coordinates of the hydrogen atom. The Heisenberg equations of motion for the dynamical variables of the hydrogen atom and the electromagnetic field can be derived from the Hamiltonian $H=H_{A}+H_{F}+H_{I}$ :

$$
\begin{aligned}
& \frac{d}{d \tau} \sigma_{m n}(\tau)=i\left(\omega_{m}-\omega_{n}\right) \sigma_{m n}(\tau)-i e \mathbf{E}(x(\tau)) \cdot\left[\mathbf{r}(\tau), \sigma_{m n}(\tau)\right], \\
& \frac{d}{d t} a_{\vec{k}}(t(\tau))=-i \omega_{\vec{k}} a_{\vec{k}}(t(\tau))-i e \mathbf{r}(\tau) \cdot\left[\mathbf{E}(x(\tau)), a_{\vec{k}}(t(\tau))\right] \frac{d \tau}{d t} .
\end{aligned}
$$

In the solutions of the equations of motion, we can separate the "free" and "source" parts,

$$
\sigma_{m n}(\tau)=\sigma_{m n}^{f}(\tau)+\sigma_{m n}^{s}(\tau), \quad a_{\vec{k}}(t(\tau))=a_{\vec{k}}^{f}(t(\tau))+a_{\vec{k}}^{s}(t(\tau))
$$

where

$$
\begin{aligned}
& \sigma_{m n}^{f}(\tau)=\sigma_{m n}^{f}\left(\tau_{0}\right) e^{i\left(\omega_{m}-\omega_{n}\right)\left(\tau-\tau_{0}\right)}, \quad \sigma_{m n}^{s}(\tau)=-i e \int_{\tau_{0}}^{\tau} d \tau^{\prime} \mathbf{E}^{f}\left(x\left(\tau^{\prime}\right)\right) \cdot\left[\mathbf{r}^{f}\left(\tau^{\prime}\right), \sigma_{m n}^{f}(\tau)\right], \quad(7) \\
& a_{\vec{k}}^{f}(t(\tau))=a_{\vec{k}}^{f}\left(t\left(\tau_{0}\right)\right) e^{-i \omega_{\vec{k}}\left[t(\tau)-t\left(\tau_{0}\right)\right]}, \quad a_{\vec{k}}^{s}(t(\tau))=-i e \int_{\tau_{0}}^{\tau} d \tau^{\prime} \mathbf{r}^{f}\left(\tau^{\prime}\right) \cdot\left[\mathbf{E}^{f}\left(x\left(\tau^{\prime}\right)\right), a_{\vec{k}}^{f}(t(\tau))\right] .
\end{aligned}
$$




\section{THE CONTRIBUTIONS OF VACUUM FLUCTUATION AND RADIATION REACTION}

We now generalize the formalism of Ref. 7] to the model described in the proceeding section. We assume that the initial state of the field is the vacuum $|0\rangle$, while the atom is in the state $|b\rangle$. The equation of motion in the interaction representation for an arbitrary atomic observable $O(\tau)$, using symmetric ordering [5], can be separated in the vacuum fluctuations and the reaction field contributions,

$$
\frac{d O(\tau)}{d \tau}=\left(\frac{d O(\tau)}{d \tau}\right)_{V F}+\left(\frac{d O(\tau)}{d \tau}\right)_{R R},
$$

where

$$
\left(\frac{d O(\tau)}{d \tau}\right)_{V F}=-\frac{i e}{2}\left(\mathbf{E}^{f}(x(\tau)) \cdot[\mathbf{r}(\tau), O(\tau)]+[\mathbf{r}(\tau), O(\tau)] \cdot \mathbf{E}^{f}(x(\tau))\right)
$$

representing the contribution of the vacuum fluctuations and

$$
\left(\frac{d O(\tau)}{d \tau}\right)_{R R}=-\frac{i e}{2}\left(\mathbf{E}^{s}(x(\tau)) \cdot[\mathbf{r}(\tau), O(\tau)]+[\mathbf{r}(\tau), O(\tau)] \cdot \mathbf{E}^{s}(x(\tau))\right),
$$

denoting that of the radiation reaction.

Our purpose now is to identify the contributions of vacuum fluctuations and radiation reaction in the evolution of the atom's excitation energy, which is given by the expectation value of $H_{A}$. Separating $r_{i}(\tau)$ and $\sigma_{n n}(\tau)$ into their free part and source part and taking the vacuum expectation value, we can obtain, in a perturbation treatment up to order $e^{2}$,

$$
\begin{gathered}
\left\langle 0\left|\frac{d H_{A}(\tau)}{d \tau}\right| 0\right\rangle_{V F}=-e^{2} \int_{\tau_{0}}^{\tau} d \tau^{\prime} C_{i j}^{F}\left(x(\tau), x\left(\tau^{\prime}\right)\right)\left[r_{j}^{f}\left(\tau^{\prime}\right),\left[r_{i}^{f}(\tau), \sum_{n} \omega_{n} \sigma_{n n}^{f}(\tau)\right]\right], \\
\left\langle 0\left|\frac{d H_{A}(\tau)}{d \tau}\right| 0\right\rangle_{R R}=e^{2} \int_{\tau_{0}}^{\tau} d \tau^{\prime} \chi_{i j}^{F}\left(x(\tau), x\left(\tau^{\prime}\right)\right)\left\{r_{j}^{f}\left(\tau^{\prime}\right),\left[r_{i}^{f}(\tau), \sum_{n} \omega_{n} \sigma_{n n}^{f}(\tau)\right]\right\} .
\end{gathered}
$$

The statistical functions $C_{i j}^{F}$ and $\chi_{i j}^{F}$ of the field are defined as

$$
\begin{aligned}
C_{i j}^{F} & =\frac{1}{2}\left\langle 0\left|\left\{E_{i}^{f}(x(\tau)), E_{j}^{f}\left(x\left(\tau^{\prime}\right)\right)\right\}\right| 0\right\rangle, \\
\chi_{i j}^{F} & =\frac{1}{2}\left\langle 0\left|\left[E_{i}^{f}(x(\tau)), E_{j}^{f}\left(x\left(\tau^{\prime}\right)\right)\right]\right| 0\right\rangle,
\end{aligned}
$$

$C_{i j}^{F}$ and $\chi_{i j}^{F}$ are the symmetric correlation function and linear susceptibility of the field in the vacuum state.

We are interested in the evolution of expectation values of atomic observables, so we take the expectation value of Eqs.(12) and (13) in the atom's state $|b\rangle$. Using the Heisenberg 
equation of motion, we can replace the commutator $\left[r_{i}^{f}(\tau), \sum_{n} \omega_{n} \sigma_{n n}^{f}(\tau)\right]$ with $i \frac{d}{d \tau} r_{i}^{f}(\tau)$, and obtain

$$
\begin{aligned}
& \left\langle\frac{d H_{A}(\tau)}{d \tau}\right\rangle_{V F}=2 i e^{2} \int_{\tau_{0}}^{\tau} d \tau^{\prime} C_{i j}^{F}\left(x(\tau), x\left(\tau^{\prime}\right)\right) \frac{d}{d \tau}\left(\chi_{i j}^{A}\right)_{b}\left(\tau, \tau^{\prime}\right), \\
& \left\langle\frac{d H_{A}(\tau)}{d \tau}\right\rangle_{R R}=2 i e^{2} \int_{\tau_{0}}^{\tau} d \tau^{\prime} \chi_{i j}^{F}\left(x(\tau), x\left(\tau^{\prime}\right)\right) \frac{d}{d \tau}\left(C_{i j}^{A}\right)_{b}\left(\tau, \tau^{\prime}\right),
\end{aligned}
$$

where |\rangle$=|b, 0\rangle$. Here symmetric correlation function and linear susceptibility of the atom are defined analogously to Eqs.(14) and (15) as

$$
\begin{aligned}
\left(C_{i j}^{A}\right)_{b}\left(\tau, \tau^{\prime}\right) & =\frac{1}{2}\left\langle b\left|\left\{r_{i}^{f}(\tau), r_{j}^{f}\left(\tau^{\prime}\right)\right\}\right| b\right\rangle, \\
\left(\chi_{i j}^{A}\right)_{b}\left(\tau, \tau^{\prime}\right) & =\frac{1}{2}\left\langle b\left|\left[r_{i}^{f}(\tau), r_{j}^{f}\left(\tau^{\prime}\right)\right]\right| b\right\rangle .
\end{aligned}
$$

They do not depend on the trajectory of the atom but characterize only the atom itself. The explicit forms of the statistical functions of the atom are given by

$$
\begin{aligned}
\left(C_{i j}^{A}\right)_{b}\left(\tau, \tau^{\prime}\right) & =\frac{1}{2} \sum_{d}\left[\left\langle b\left|r_{i}(0)\right| d\right\rangle\left\langle d\left|r_{j}(0)\right| b\right\rangle e^{i \omega_{b d}\left(\tau-\tau^{\prime}\right)}+\left\langle b\left|r_{j}(0)\right| d\right\rangle\left\langle d\left|r_{i}(0)\right| b\right\rangle e^{-i \omega_{b d}\left(\tau-\tau^{\prime}\right)}\right], \\
\left(\chi_{i j}^{A}\right)_{b}\left(\tau, \tau^{\prime}\right) & =\frac{1}{2} \sum_{d}\left[\left\langle b\left|r_{i}(0)\right| d\right\rangle\left\langle d\left|r_{j}(0)\right| b\right\rangle e^{i \omega_{b d}\left(\tau-\tau^{\prime}\right)}-\left\langle b\left|r_{j}(0)\right| d\right\rangle\left\langle d\left|r_{i}(0)\right| b\right\rangle e^{-i \omega_{b d}\left(\tau-\tau^{\prime}\right)}\right],
\end{aligned}
$$

where $\omega_{b d}=\omega_{b}-\omega_{d}$ and the sum extends over a complete set of atomic states.

In order to get the statistical functions for the field, we will use following the two point function for the photon field in the Feynman gauge

$$
\left\langle 0\left|A_{\mu}(x) A_{\nu}\left(x^{\prime}\right)\right| 0\right\rangle=\frac{\eta_{\mu \nu}}{4 \pi^{2}\left[\left(t-t^{\prime}-i \varepsilon\right)^{2}-\left(x-x^{\prime}\right)^{2}-\left(y-y^{\prime}\right)^{2}-\left(z-z^{\prime}\right)^{2}\right]},
$$

so, the field correlation function can be calculated as follows

$$
\left\langle 0\left|E_{i}(x(\tau)) E_{j}\left(x\left(\tau^{\prime}\right)\right)\right| 0\right\rangle=\frac{1}{4 \pi^{2}}\left(\partial_{0} \partial_{0}^{\prime} \delta_{i j}-\partial_{i} \partial_{j}^{\prime}\right) \frac{1}{\left(x-x^{\prime}\right)^{2}+\left(y-y^{\prime}\right)^{2}+\left(z-z^{\prime}\right)^{2}-\left(t-t^{\prime}-i \varepsilon\right)^{2}},
$$

where $\varepsilon \rightarrow+0$ and $\partial^{\prime}$ denotes the differentiation with respect to $x^{\prime}$.

\section{UNIFORMLY ACCELERATED ATOM}

Let us now apply the formalism just developed to study the spontaneous emission of the atom which is uniformly accelerated in the $x$-direction. Specifically, the atom's trajectory is described by

$$
t(\tau)=\frac{1}{a} \sinh a \tau, \quad x(\tau)=\frac{1}{a} \cosh a \tau, \quad y(\tau)=z(\tau)=0 .
$$


The field correlation function for the trajectory (24) can be evaluated from its general form (23) in the frame of the atom to get

$$
\left\langle 0\left|E_{i}(x(\tau)) E_{j}\left(x\left(\tau^{\prime}\right)\right)\right| 0\right\rangle=\delta_{i j} \frac{1}{16 \pi^{2}} \frac{a^{4}}{\sinh ^{4}\left[\frac{a}{2}\left(\tau-\tau^{\prime}-i \varepsilon\right)\right]} .
$$

From Eq.(25), we obtain the symmetric correlation function

$$
C_{i j}^{F}\left(x(\tau), x\left(\tau^{\prime}\right)\right)=\delta_{i j} \frac{a^{4}}{32 \pi^{2}}\left(\frac{1}{\sinh ^{4}\left[\frac{a}{2}\left(\tau-\tau^{\prime}-i \varepsilon\right)\right]}+\frac{1}{\sinh ^{4}\left[\frac{a}{2}\left(\tau-\tau^{\prime}+i \varepsilon\right)\right]}\right)
$$

and the linear susceptibility

$$
\chi_{i j}^{F}\left(x(\tau), x\left(\tau^{\prime}\right)\right)=-i \delta_{i j} \frac{1}{\pi\left(\cosh ^{3} \frac{a\left(\tau-\tau^{\prime}\right)}{2}+5 \cosh \frac{a\left(\tau-\tau^{\prime}\right)}{2}\right)} \delta^{(3)}\left(\tau-\tau^{\prime}\right),
$$

where $\delta^{(3)}$ is the third derivative of the Dirac delta function [13]. With a substitution $u=\tau-\tau^{\prime}$, we get from (17)

$$
\left\langle\frac{d H_{A}(\tau)}{d \tau}\right\rangle_{R R}=\frac{i e^{2}}{\pi} \sum_{d} \omega_{b d}|\langle b|\mathbf{r}(0)| d\rangle|^{2} \times \int_{-\infty}^{\infty} d u \frac{e^{i \omega_{b d} u}}{\cosh ^{3} \frac{a u}{2}+5 \cosh \frac{a u}{2}} \delta^{(3)}(u) .
$$

Here, we have extend the range of integration to infinity for sufficiently long times $\tau-\tau_{0}$. After the evaluation of the integral, we get

$$
\left\langle\frac{d H_{A}(\tau)}{d \tau}\right\rangle_{R R}=-\frac{e^{2}}{6 \pi}\left(\sum_{\omega_{d}<\omega_{b}} \omega_{b d}^{4}|\langle b|\mathbf{r}(0)| d\rangle|^{2}\left(\frac{a^{2}}{\omega_{b d}^{2}}+1\right)+\sum_{\omega_{d}>\omega_{b}} \omega_{b d}^{4}|\langle b|\mathbf{r}(0)| d\rangle|^{2}\left(\frac{a^{2}}{\omega_{b d}^{2}}+1\right)\right) .
$$

The rate of change of the atomic energy is corrected by the acceleration as compared to the inertial case $(a=0)$ and it always leads to a loss of energy of the atoms. In contrast to the scalar field case [7], where the uniform acceleration does not change the contribution of the radiation reaction, in our present case, there is an extra correction proportional to $a^{2}$. The contribution of the vacuum fluctuation to the rate of the atomic energy becomes now

$$
\left\langle\frac{d H_{A}(\tau)}{d \tau}\right\rangle_{V F}=-\frac{e^{2} a^{4}}{32 \pi^{2}} \sum_{d} \omega_{b d}|\langle b|\mathbf{r}(0)| d\rangle|^{2} \times \int_{-\infty}^{\infty} d u\left(\frac{1}{\sinh ^{4}\left[\frac{a}{2}(u-i \varepsilon)\right]}+\frac{1}{\sinh ^{4}\left[\frac{a}{2}(u+i \varepsilon)\right]}\right) e^{i \omega_{b d} u}
$$

One can calculate Eq.(30) by residues to get

$$
\begin{aligned}
\left\langle\frac{d H_{A}(\tau)}{d \tau}\right\rangle_{V F}= & -\frac{e^{2}}{6 \pi}\left(\sum_{\omega_{d}<\omega_{b}} \omega_{b d}^{4}|\langle b|\mathbf{r}(0)| d\rangle|^{2}\left(\frac{a^{2}}{\omega_{b d}^{2}}+1\right)\left(1+\frac{2}{e^{\frac{2 \pi \omega_{b d}}{a}}-1}\right)\right. \\
& \left.-\sum_{\omega_{d}>\omega_{b}} \omega_{b d}^{4}|\langle b|\mathbf{r}(0)| d\rangle|^{2}\left(\frac{a^{2}}{\omega_{b d}^{2}}+1\right)\left(1+\frac{2}{e^{\frac{2 \pi\left|\omega_{b d}\right|}{a}}-1}\right)\right) .
\end{aligned}
$$


This result reveals that vacuum fluctuations equally lead to excitation of an accelerated ground-state atom and de-excitation of an excited one and the probabilities of these two processes are enhanced by the acceleration dependent correction terms as compared to the inertial case. The most distinct feature in the present case in contrast to that of a scalar field is that in addition to a thermal term, there exists an extra correction proportional to $a^{2}$. The extra term is not in the form of a thermal effect. This is in sharp contrast with the scalar field case [7] where the effect of acceleration upon the contribution of vacuum fluctuations is only a "thermal" correction with the temperature $T=a / 2 \pi$. Therefore, one sees that the equivalence between uniform acceleration and thermal field is lost in the present case. For a typical transition frequency in the Pfund Series of a hydrogen atom, $\omega \sim 10^{13} \mathrm{~s}^{-1}$, the nonthermal correction would be comparable to the thermal effect when the acceleration is approximately $\sim \omega \sim 10^{23} \mathrm{~cm} / \mathrm{s}^{2}$. This acceleration, although extremely large, is at least of the same order of the acceleration necessary to observe the thermal effect associated with uniform acceleration in atomic systems [12].

Finally, we add the contributions of vacuum fluctuations (29) and radiation reaction (31) to obtain the total rate of change of the atomic excitation energy:

$$
\begin{aligned}
\left\langle\frac{d H_{A}(\tau)}{d \tau}\right\rangle_{t o t}= & -\frac{e^{2}}{3 \pi}\left(\sum_{\omega_{d}<\omega_{b}} \omega_{b d}^{4}|\langle b|\mathbf{r}(0)| d\rangle|^{2}\left(\frac{a^{2}}{\omega_{b d}^{2}}+1\right)\left(1+\frac{1}{e^{\frac{2 \pi \omega_{b d}}{a}}-1}\right)\right. \\
& \left.-\sum_{\omega_{d}>\omega_{b}} \omega_{b d}^{4}|\langle b|\mathbf{r}(0)| d\rangle|^{2}\left(\frac{a^{2}}{\omega_{b d}^{2}}+1\right) \frac{1}{e^{\frac{2 \pi\left|\omega_{b d}\right|}{a}}-1}\right) .
\end{aligned}
$$

For a uniformly accelerated atom in its ground state, only the second term contributes. This ensures the stability of ground-state atom at one hand and allows the transition to the excited states of accelerated ground-state atoms in the vacuum on the other.

\section{CONCLUSIONS}

In conclusion, assuming a linear coupling between a multi-level atom and a quantum electromagnetic field, we have studied the contributions of vacuum fluctuations and radiation reaction to the rate of change of the atomic energy of a uniformly accelerated atom. If the atom moves with constant acceleration, the perfect balance between the contributions of vacuum fluctuations and radiation reaction that ensures the stability of ground-state atoms is disturbed, making spontaneous transition of ground-state atoms to excited states possible. We find that the effects of electromagnetic vacuum fluctuations on the spontaneous rate of an accelerated atom is not purely thermal with the temperature $T=a / 2 \pi$, and there exists a non-thermal correction, which might be observable. This is in sharp contrast to the scalar field case [7]. Another interesting feature worth noting, in contrast to the scalar field case 7], is that the the acceleration also affects the contribution of radiation reaction to the rate of change of atomic energy. 


\section{Acknowledgments}

This work was supported in part by the National Natural Science Foundation of China under Grants No.10375023 and No.10575035, the Program for NCET (No.04-0784), the Key Project of Chinese Ministry of Education (No.205110) and the Research fund of Hunan Provincial Education Department (No. 04A030)

[1] T. A. Welton, Phys. Rev. 74,1157(1948).

[2] G. Compagno, R. Passante and F. Persico, Phys. Lett. A 98,253(1983).

[3] J. R. Ackerhalt, P. L. Knight and J. H. Eberly, Phys. Rev. Lett. 30, 456(1973).

[4] P. W. Milonni, Phys. Scr. 21, 102(1988); P. W. Milonni and W. A. Smith, Phys. Rev. A 11, 814(1975).

[5] J. Dalibard, J. dupont-Roc and C. Cohen-Tannodji, J. Physique 43, 1617(1982).

[6] J. Dalibard, J. dupont-Roc and C. Cohen-Tannodji, J. Physique 45, 637(1984).

[7] J. Audretsch and R. Müller, Phys. Rev. A 50 1755(1994); Class. Quant. Grav. 12, 2927(1995).

[8] J. Audretsch and R. Müller, Phys. Rev. A 52, 629(1995).

[9] H. Yu and S. Lu, Phys. Rev. D 72, 064022(2005).

[10] R. Passante, Phys. Rev. A 57, 1590(1997).

[11] T.H. Boyer, Phys. Rev. D 21, 2137(1980).

[12] For a recent review on various kinds of clever proposals, see H. C. Rosu, e-print hep-th/0301128.

[13] Let us note that there is an error in Eq. (3.4) in Ref. [10] 